Int. J. Curr. Res. Med. Sci. (2017). 3(7): 24-29

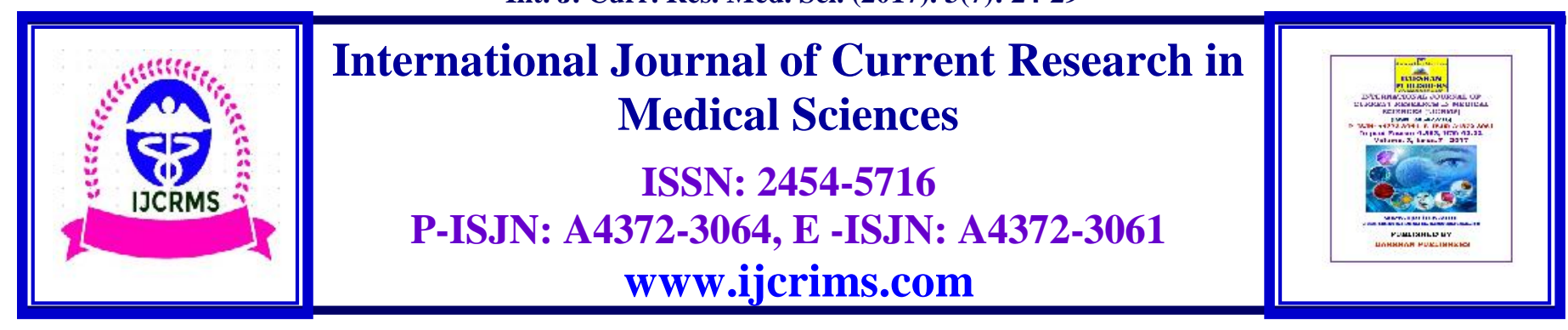

\title{
To study haematological profile of Enteric fever patients.
}

\author{
Dr SPS Dhillon ${ }^{1}$, Dr Neeraj Lata ${ }^{2}$, Dr Sehajpreet Singh ${ }^{3}$, Dr Vijay Gotwal ${ }^{4}$, \\ Dr Tanushree Joshi ${ }^{5}$, Dr Narinder Singh ${ }^{6}$ \\ ${ }^{1}$ Assistant Professor, Department of Pediatrics, Government College Amritsar, Punjab \\ ${ }^{2}$ Senior Resident, Department of Pediatrics, Government Medical College, Amritsar, Punjab \\ ${ }^{3}$ Intern, Vardhman Mahavir Medical College , and Safdarjung Hospital, New Delhi \\ ${ }^{4}$ Junior Resident, Department of Chest and Tuberculosis, Government Medical College, Amritsar, Punjab \\ ${ }^{5}$ SeniorDMO, Northern Railway Central Hospital, New Delhi \\ ${ }^{6}$ Assistant Professor, Department of Pediatrics, Government College Amritsar, Punjab \\ Corresponding author: Dr Neeraj Lata \\ Senior Resident, Department of Pediatrics, Government Medical College, Amritsar \\ E-mail id:neerajlatavijay@gmail.com
}

\begin{abstract}
Introduction: Typhoid fever is a major health problem in developing countries. It is caused by Salmonella typhi and $S$. paratyphi .In India annual incidence is nearly $1 \%$.Blood culture for Salmonella is the gold standard to diagnose typhoid fever. Hematological profile of these patients is affected by this infection. We took this study to establish the significance of hematological profile in blood culture proven Typhoid fever patients.

Material and Methods : We did a cross sectional study on 100 patients attending OPD and IPD of Department of Pediatrics, with history of fever of $>38^{\circ}$ Cfor 3 days aged 1-14 years. Previously antibiotic treated patients and patients with proven localised infection were excluded .Panel of tests including blood culture and haematological profile (Hemoglobin, TLC ,DLC, Platelet count, ESR) were done on these patients .

Results: In our study $46 \%$ of our patients were blood culture proven typhoid fever patients. Male children of school going age group were most commonly affected by typhoid fever. We found anemia, leucopenia, thrombocytopenia in $47.8 \%, 6.5 \%$ and $21.5 \%$ of patients. Lymphocytosis was seen in $10.9 \%$ of patients.ESR was high in $52.2 \%$ of patients. Results were comparable to studies done in past but we found that out of all these parameters only ESR was significantly associated with blood culture positivity.

Conclusion : Anemia in typhoid fever patients is not because of the salmonella infection .It can be because of other reasons for which detailed blood indices are required to be investigated .Total leucocyte count, eosinophil count and platelet count are also not contributory to diagnosis of enteric fever. High ESR is associated with typhoid fever. Further studies are needed to study the blood indices in detail in patients with typhoid fever in India.
\end{abstract}

Keywords: Typhoid fever, Hematological profile, leucopenia 


\section{Introduction}

The term enteric fever includes typhoid fever caused by S.typhi and paratyphoid fever caused by S.paratyphi A, B and C. Detailed study of enteric fever was presented by Bretonneau (1826) who identified intestinal lesions . The name typhoid fever was given by Louis (1829) to distinguish it from typhus fever. Eberth (1880) described typhoid bacillus . ${ }^{1}$ According to WHO Confirmed case of typhoid fever is defined, as a patient with fever $\left(>38^{\circ} \mathrm{C}\right)$ that has lasted for at least three days, with a laboratory confirmed positive culture of S. typhi. ${ }^{2}$ Probable case of typhoid fever is a patient with fever $\left(>38^{\circ} \mathrm{C}\right)$ that has lasted for $>3$ days, with a positive serodiagnosis or antigen detection test but without S. typhi isolation. ${ }^{2}$ The world sees approximately 22 million new typhoid cases occur each year ${ }^{6}$. In India in disease-endemic areas, the annual incidence of enteric fever is about $1 \%{ }^{3}$ Complete blood counts in enteric fever are found to be unremarkable. The hemoglobin is normal in the initial stages but drops with progressing illness. Severe anemia is unusual and should make one suspect intestinal hemorrhage or hemolysis or an alternative diagnosis like malaria. The WBC count is normal in most cases and leucocytosis makes the diagnosis less probable. Leukopenia is perceived to be an important feature of typhoid fever and has been reported in only $20-25 \%$ cases. ${ }^{4}$ The differential count is usually unremarkable except for eosinopenia. Eosinopenia often absolute may be present in $70-80 \%$ cases. ${ }^{5}$ Presence of absolute eosinopenia offers a clue to diagnosis but does not differentiate enteric fever from other acute bacterial or viral infections. A normal eosinophil count does make typhoid fever a less likely possibility. Platelet counts are normal to begin with and fall in some cases by the second week of illness. Overall prevalence of thrombocytopenia is around $10-15 \%{ }^{4}$. We did this study to evaluate the blood indices in culture proven enteric fever patients.

\section{Aims and objectives}

To study haematological profile of Typhoid fever patients.

\section{Study design}

The present study was a hospital based crosssectional study. 100 Patients with fever were evaluated by doing a panel of tests for typhoid fever and complete blood count of these patients were done.

\section{Inclusion criteria}

1. Age:1-14yrs

2. Both sexes

3. Both Indoor and outdoor patients

4. Duration of fever of more >3days.

5. Fever $>38^{\circ} \mathrm{C}$

\section{Exclusion criteria}

1. Previously antibiotic treated patients.

2. Proven localized infection.

\section{Materials and Methods}

100 patients from opd and indoor admission with fever of greater than 3 days duration were included in the study. Complete blood count ,blood culture for typhoid fever and other tests to rule out other causes of fever were done .Blood culture of these patients was done by BacT/ALERT Microbial Detection System. Hemoglobin, TLC, DLC, Eosinophil Count, Platelet count, and ESR samples were sent to the biochemistry department .

\section{Results}

Total of 100 Patients with fever for more than 3 days attending OPD and IPD were evaluated by doing blood culture and blood counts (Hemoglobin, TLC, DLC, Eosinophil count, Platelet count, ESR) .

Demographic, clinical data, laboratory Parameter details were noted and analysed using SPSS software version 17(SPSS Inc., Chicago, IL, USA). 
Int. J. Curr. Res. Med. Sci. (2017). 3(7): 24-29

Table1:Gender distribution of patients

\begin{tabular}{|ll|l|l|l|l|}
\hline & & Frequency & Percent & Valid Percent & $\begin{array}{l}\text { Cumulative } \\
\text { Percent }\end{array}$ \\
\hline Valid & FEMALE & 43 & 43.0 & 43.0 & 43.0 \\
& MALE & 57 & 57.0 & 57.0 & 100.0 \\
Total & 100 & 100.0 & 100.0 & \\
\hline
\end{tabular}

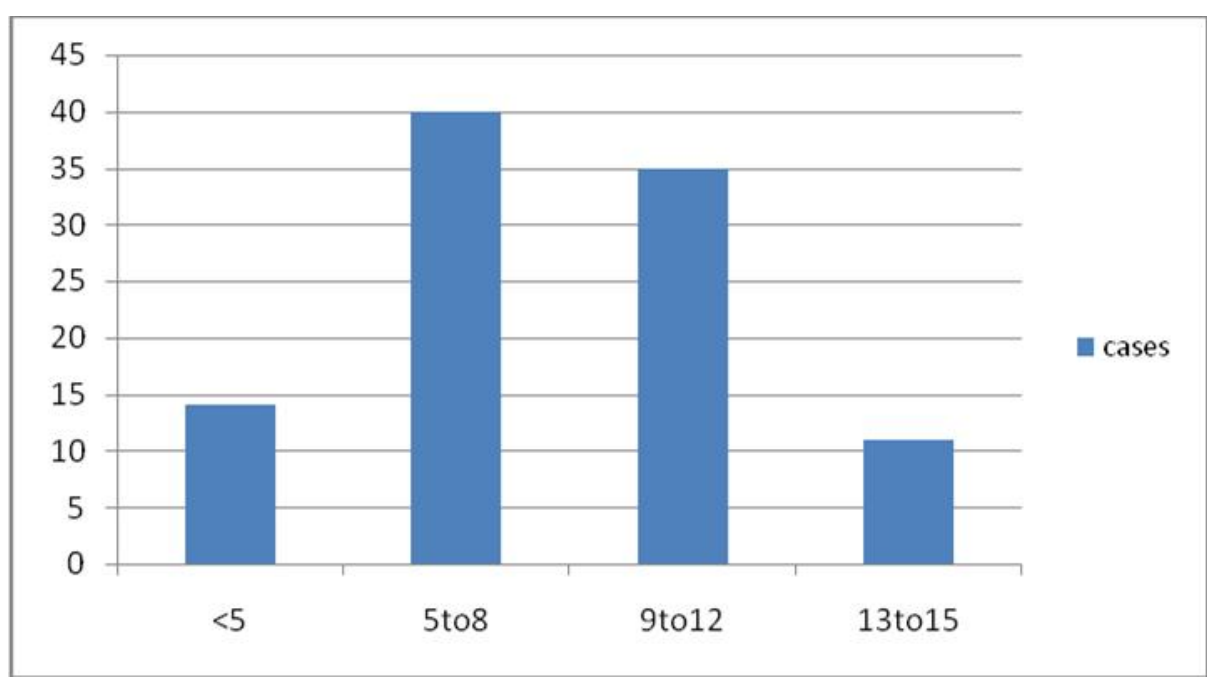

Fig 1:Age wise distribution of patients

In our study age ranged from 1.5 yrs to 14 yrs with mean age of $8.2 \mathrm{yrs}+/-3.3 \mathrm{SD}$.

Patients in age group 5-8yrs were maximally affected by typhoid fever followed by 9-12 yr age group.
In this age group males were affected more than the females.Blood culture positivity ranged from 1.5 yrs to 14 yrs with mean age $7.880+-3.24$ SD and its association with age distribution is found to be statistically not significant $(p>0.05)$.

Table2 : Frequency table for Blood culture test.

\begin{tabular}{|c|c|c|c|c|c|}
\hline & & Frequency & Percent & Valid Percent & Cumulative Percent \\
\hline \multirow[t]{3}{*}{ Valid } & POSITIVE & 46 & 46.0 & 46.0 & 46.0 \\
\hline & $\begin{array}{l}\text { NEGATIV } \\
\text { E }\end{array}$ & 54 & 54.0 & 54.0 & 100.0 \\
\hline & Total & 100 & 100.0 & 100.0 & \\
\hline
\end{tabular}

When we compared blood culture results in both genders then blood culture positivity in male gender was found to be statistically significant $(\mathrm{p}<0.05)$ 
Int. J. Curr. Res. Med. Sci. (2017). 3(7): 24-29

Table 3:Hematological test results in Blood culture Positive and negative patients

\begin{tabular}{|l|l|l|l|}
\hline Blood tests & $\begin{array}{l}\text { Blood culture } \\
\text { positive }\end{array}$ & $\begin{array}{l}\text { Blood culture } \\
\text { negative }\end{array}$ & P value \\
\hline Hb<11gm\% & $22(47.8 \%)$ & $29(53.7 \%)$ & .558 \\
\hline $\begin{array}{l}\text { Leucopenia(TLC }<40 \\
\text { 00) }\end{array}$ & $3(6.5 \%)$ & $3(5.6 \%)$ & .598 \\
\hline $\begin{array}{l}\text { Lymphocytosis } \\
(\text { TLC }>11000)\end{array}$ & $5(10.9 \%)$ & $3(5.6 \%)$ & .598 \\
\hline $\begin{array}{l}\text { Eosinophil } \\
\text { count(<1\%) }\end{array}$ & $3(6.5 \%)$ & $2(3.7 \%)$ & .763 \\
\hline $\begin{array}{l}\text { Thrombocytopenia } \\
\text { (Platelet } \\
\text { count<1.5lac) }\end{array}$ & $10(21.7 \%)$ & $10(18.55)$ & .688 \\
\hline ESR>20 & $24(52.2 \%)$ & $39(72.2 \%)$ & .038 \\
\hline
\end{tabular}

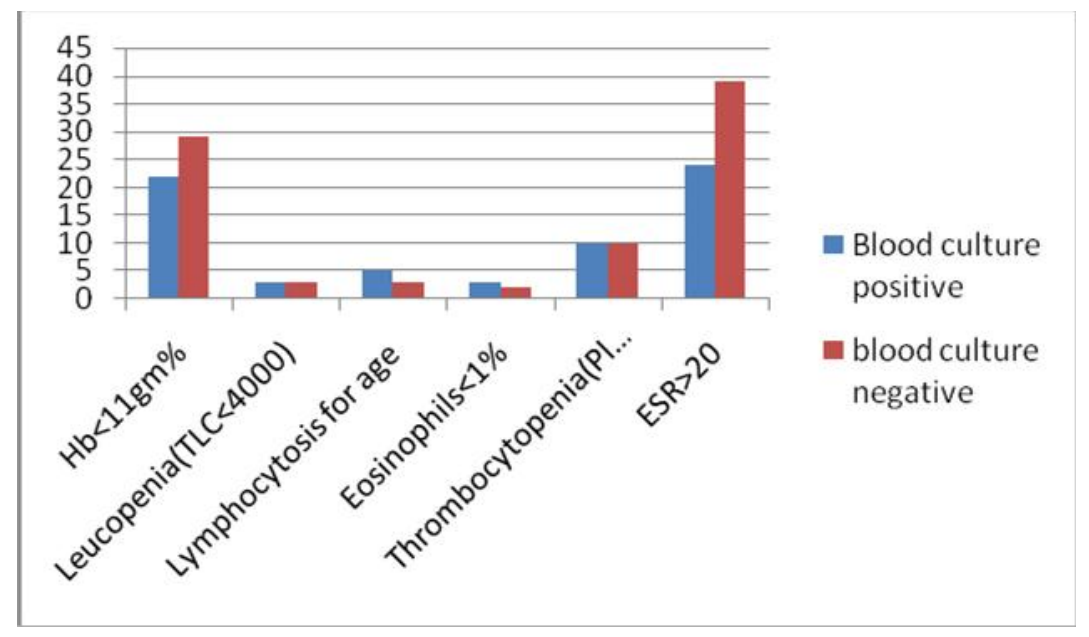

Fig 2: Graph showing the haematological indices

\section{Discussion}

A total of 100 patients of age group1-14 years of both gender were studied, who attended OPD and IPD with 3 or more days of fever of $>38 \mathrm{C}$ as per inclusion criteria .Previously antibiotic treated patients and patients with localised infection were excluded from the study. This study was carried out for a period of one year. Out of 100 patients 57patients were male and 43 were female. In our study group Male to female ratio is 1.3:1 i.e. males are more affected than females. $40 \%$ patients belonged to age group5-8 years followed by $35 \%$ in $9-12$ yrs and $14 \%$ were
$<5 y r s$, with range of 1.5-14 yrs and mean age of presentation 8.2+/-3.3SD.

Amongst the definitive cases of typhoid fever i.e. Blood culture positive for S.typhi, 39\% belonged to $5-8 \mathrm{yr}$ age group followed by $37 \%$ in $9-12 \mathrm{yr}$ and $15.2 \%$ in <5year age group. Age distribution of definite and probable cases of typhoid fever was maximum in 5-8 yrs followed by 9-12 yrs .Similar results are seen in earlier studies that typhoid fever is prevalent in 2-5 and school going children aged 5-15 yrs ${ }^{6,7}$. In our study, $15.2 \%$ of all patients with typhoid fever were under 5 years, which is close to the figure in other studies. ${ }^{9,10,18}$ 
Blood culture was positive in $46 \%$ of cases which was comparable to other studies by Bhutta et al ${ }^{11}$, Dheer et al ${ }^{13}$ and Sanjeev et al. ${ }^{15}$ Krishna et aL showed 64\%blood culture positivity in their trials. $^{16}$

Hemoglobin is normal in the initial stages but drops with progressing illness. Kakaria et al reported Anemia in $42.9 \%$ in their prospective study. Shilpa et al observed anemia in $34 \%$ of their enteric fever patients. ${ }^{12}$ Our results of anemia were comparable to these studies. Severe anemia is unusual in typhoid fever and should make one suspect intestinal haemorrhage or hemolysis or an alternative diagnosis like malaria. ${ }^{17,18}$ our Results for anemia were comparable to these studies but they were statistically not significant. It has been attributed to the maturational arrest of the cell lines in salmonella infection in bone marrow.

$6.5 \%$ of our blood culture positive patients had leucopenia. Kakaria et al reported leucopenia in $10 \%$ of their patients with typhoid fever. ${ }^{19}$ Dheer et al also reported leucopenia in $7.5 \%$ of typhoid fever patients. ${ }^{13}$ Most of our cases had normal total leucocyte counts similar to earlier reports. $11,17,18$.

Eosinopenia was seen in $6.5 \%$ of patients of definitive typhoid fever. It is seen that eosinopenia often absolute may be present in 70$80 \%$ of cases. ${ }^{4,5}$ Presence of absolute eosinopenia offers a clue to diagnosis but does not differentiate it from other acute bacterial or viral infections. ${ }^{11,17,18}$ In Our study eosinopenia is not a significant finding.

Thrombocytopenia was seen in $21.7 \%$ of blood culture positive patients of typhoid fever in our study. Dheer et al reported thrombocytopenia in $29.9 \%$ cases. $^{13}$ It is seen that overall prevalence of thrombocytopenia is around 10-15\%. ${ }^{18}$ I Nayyar et al had seen Isolated thrombocytopenia in $43 \%$ of cases typhoid fever in their study. ${ }^{14}$ Shilpa et al had shown thrombocyto penia in $17.24 \%$ of patients with enteric fever in their trial. ${ }^{12}$ Srivastava et al observed Thrombocytopenia in $39.7 \%$ in their study . ${ }^{7}$ Malik et al found thrombocytopenia in $26 \%$ of typhoid fever cases in their trial. ${ }^{8}$ Our results were comparable to these studies but it was not statistically significant.

ESR was high in $52.2 \%$ of blood culture positive and $72.2 \%$ in blood culture negative patients and it is statistically significantly associated with blood culture positivity. Darton and Cames et al had shown significant association of raised ESR in typhoid fever patients. ${ }^{3,20}$

\section{Conclusion}

Anemia in typhoid fever patients is not because of the salmonella infection .It can be because of other reasons for which detailed blood indices are required to be investigated. Total leucocyte count, eosinophil count and platelet count are also not contributory to diagnosis of enteric fever. High ESR is associated with typhoid fever. Further studies are needed to study the blood indices in patients with typhoid fever in India.

\section{Abbreviations}

TLC: Total Leucocyte Count

DLC: Differential Leucocyte Count

ESR: Erythrocyte Sedimentation Rate

$\mathrm{Hb}:$ Hemoglobin

\section{References}

1. Ananthanarayan R, Panikar CKJ. Text Book Of Microbiology, 9th Edition; Chennai: Orient Longman; 2013.

2. Background document: The diagnosis, treatment and prevention of typhoid fever. Communicable Disease Surveillance and Response Vaccines and Biologicals. World Health Organization [Internet] [cited 2015 April 6]. Available from: http://www.who. int/rpc/TFGuideWHO.pdf

3. Darton TC, Blohmke CJ, Pollard AJ. Typhoid epidemiology, diagnostics and the human challenge model. Curr Opin Gastroenterol 2014; 30:7-17.

4.Lokhandwala ,Syed Athar,Nicolas P Turin. Role of Absolute Eosinopenia as Marker of Enteric Fever: Experience from a Tertiary Care Hospital in the United Arab Emirates Aliasgar Ibnosina J Med BS 2012:249-253

5. Kuvandik C, Karaoglan I, Namiduru M, Baydar I. Predictive value of clinical and laboratory 
Int. J. Curr. Res. Med. Sci. (2017). 3(7): 24-29

findings in the diagnosis of the enteric fever. New Microbiol. 2009 Jan;32(1):25-30.

6. Ochiai RL, Acosta CJ, Danovaro-Holliday MC, Baiqing D, Bhattacharya SK, AgtiniMD et al.Typhoid Study Group. A study of typhoid fever in five Asian countries: disease burden and implications for controls. Bull World Health Organ 2008; 86:260-8.

7..Shrivastava K, Vahikar S, Mishra V. Hematological profile in typhoid fever. Trop J Path Micro 2015;1(1):16-20.

8..Malik AS, Malik RH. Typhoid fever in Malaysian children. Med J Malaysia. 2001 Dec;56(4):478-90.

9. Ahmet Yaramis, Idris Yildirim, Selahattin Katar, M. Nuri Özbek, Isik Yalçin, M. Ali Tas et al. Clinical and Laboratory Presentation of Typhoid Fever. International Pediatrics/Vol. 16/No. 4/2001; 227-31.

10.Brooks WA, Hossain A, Goswami D, Nahar $\mathrm{K}$, Alam K, Ahmed N. Bacteremic typhoid fever in children in an urban slum, Bangladesh. Emerg Infect Dis 2005; 11:326-9.

11. Bhutta ZA, Dewraj HL. Current concepts in the diagnosis and treatment of typhoid fever. BMJ 2006; 333:78-82.

12.Shilpa V. U, Syeda H K, Mandakini B. T. Haematological profile in typhoid fever :IJPO 2017;4(2):263-265

13. DheerG, KundraS, SinghS.Clinical and laboratory profile of enteric fever in children in northern India. Tropical doctor 2012:1-3.

14.N, Basheer A, Mookkappan S, Ramdas A, Varghese RG, Padhi S, Shrimanth B, et al.
Clinicopathological profile of salmonella typhi and paratyphi infections presenting as Fever of unknown origin in a tropical country. Mediterr J Hematol Infect Dis. 2015 Mar 1;7(1)

15 .Sanjeev H , NayakS , Pai Asha K.B. , RekhaR , Vimal K , Ganesh H.R. A Systematic evaluation of rapid dot-EIA, blood culture and Widal test in the diagnosis of typhoid fever. NUJHS 2013; 3:21-4.

16. Krishna S, Desai S, Anjana VK, Paranthaaman RG. Typhodot(IgM) as a reliable and rapid diagnostic test for typhoid fever. Ann Trop Med Pub Health 2011; 4:4244

17.Typhoid infection diagnosis - step-by-step. BMJ Best Practice [Internet] [cited 2015 April 7]. Available from: http://bestpractice. bmj.com/best-practice/monograph/221/ diagnosis/step-by-step.html

18. IAP Guidelines 2006;19-27.

19.Kakaria A, Asgaonkar D, Narkhede M. Clinical profile of enteric fever: a prospective study of fifty enteric fever patients. Int J Res Med Sci 2014;2:1620-5 Anemia, leukocytosis and leukopenia and elevated liver enzymes were found in $42.9 \%, 10 \%, 21 \%$ and $45 \%$ respectively

20. Caumes E, Nilouphar E, Jacqueline N, Francois B.Typhoid and Paratyphoid Fever: A 10-Year Retrospective Study of 41 Cases in a Parisian Hospital. J Travel Med 2001; 8:293297.

\begin{tabular}{|c|l|}
\hline \multicolumn{2}{|c|}{ Access this Article in Online } \\
\hline & Website: \\
& www.ijcrims.com \\
\hline Quick Response Code & Subject: \\
& \\
\hline
\end{tabular}

How to cite this article:

SPS Dhillon, Neeraj Lata, Sehajpreet Singh, Vijay Gotwal, Tanushree Joshi, Narinder Singh. (2017). To study haematological profile of Enteric fever patients. Int. J. Curr. Res. Med. Sci. 3(7): 24-29.

DOI: http://dx.doi.org/10.22192/ijcrms.2017.03.07.004 